\title{
LA ARQUITECTURA GRÁFICA COMO BASE ESTRATÉGICA COMPETITIVA EN EL DESARROLLO ECONÓMICO LOCAL DE CIUDADES DE FRONTERAS
}

\author{
Ender José Barrientos-Monsalve \\ dr.ender.utel@gmail.com \\ Universidad Tecnológica Latinoamericana en Línea \\ José Ascensión Cáceres-Caballero \\ cacerescaballero@gmail.com \\ Universidad de Santander \\ Javier Alfonso-Cárdenas \\ javieralfonsocg@ufps.edu.co \\ Universidad Francisco de Paula Santander
}

\section{Resumen}

Las ciudades de frontera son vistas globalmente como entes territoriales en cuya esencia proyectiva basa sus estrategias en función a la cultura turística que de ellas se emane, pero su punto cardinal de interés, parte desde la arquigrafía. El propósito general de esta investigación es describir la identidad cultural desde la perspectiva de la arquigrafía, en las ciudades fronterizas a través de su propia esencia, como una estrategia competitiva del turismo en el desarrollo económico local. Para ello, la investigación se orientó bajo un enfoque cualitativo, en la modalidad documental, de revisión bibliográfica sistematizada, centrada en el método hermenéutico. En conclusión, el sentimiento de pertenencia social está dado por las teorías antropológicas desde la perspectiva de la identidad cultural. En el contexto fronterizo, los pilares del fortalecimiento económico de la población local juegan un papel importante, con la variabilidad, la adaptabilidad, la sustentabilidad y la sostenibilidad que representa la importancia de la arquigrafía. En sí misma, la promoción de la identidad cultural es, arquigráficamente hablando, una palanca económica de la competencia en su fortalecimiento, y el estímulo turístico de los mercados, que repercuten en el desarrollo local de las ciudades fronterizas, bajo el procedimiento adecuado de la identificación del tejido empresarial que se despliega desde allí. 
Introducción

La identidad cultural viene representada como aquel conjunto de valores, tradiciones, símbolos, creencias y modos de comportamiento que funcionan como elemento cohesionador dentro de un grupo social en determinado territorio, es decir, lo espacial y temporalidad, son asumidos como acciones de fundamento para que los individuos al formarse puedan fundamentar su sentimiento de pertenencia social en determinado lugar territorial, y de allí promocionar turísticamente su estructura social de mercados.

Como antesala al estudio, global del tema de la identidad cultural, se debe abordar los Objetivos del Desarrollo de Milenio, pues, se encuentra que su tendencia está en fomentar metas sobre las necesidades de países que, de alguna manera, se encuentran limitados en el desarrollo comercial, financiero, e incluso en nuevas tecnologías de la información y la comunicación; su fin es abordar la reducción del hambre y la mejora de la agricultura y la seguridad alimentaria.

Entonces, se hace necesario el efecto dinámico de la seguridad alimentaria, a través de políticas comerciales globales, entre ellas la asistencia técnica, el intercambio de buenas prácticas por medio de redes en el campo, capacitación en gestión y uso de las tecnologías modernas. Si bien todo esto es parte de un desarrollo local, las metas que se pretenden atomizan un sistema comercial y financiero abierto, las necesidades de países menos adelantados y con desarrollo sin litoral y los pequeños Estados insulares en desarrollo, la deuda de los países en vías de desarrollo, el acceso a los medicamentos en países en desarrollo 
a precios asequibles; y finalmente, el acceso a beneficios de las nuevas tecnologías (Organización de las Naciones Unidas para la Alimentación y Agricultura, 2020).

Como puede observarse, los objetivos de desarrollo del milenio concuerdan en que, el desarrollo en ciudades de fronteras, por condiciones especiales, también requiere su atención, pues, desde ellas se vislumbran las potencialidades en función a una cultura de identidad en el desarrollo económico local.

El ámbito de la identidad cultural, desde la óptica promocional de ésta, interviene el enfoque de las teorías antropológicas, la cual, incursiona en las dinámicas internas de desarrollo de las sociedades particulares, pues, los factores externos correlacionan las fuerzas entre la estabilidad y el cambio. Por esa razón, el sistema y filtros culturales de las ciudades de frontera deben ser reinterpretados, cuando todo lo que llega desde el exterior es diferente al típico o local, y traen cosas nuevas y actúan diferentes. En esencia, la historia ha demostrado que los sistemas sociales locales, también se desarrollan en el contexto antropológico y etnológico desde la reflexión de la identidad cultural, para motivar la promocionalidad de su esencia y su contextualidad.

Las ciudades de fronteras son entidades sociales creadas en el espacio territorial a través del tiempo, que comprende y cobija las diferencias dentro de sus límites, es decir, una comunidad que se conforma por individuos activos que comparten un mismo espacio, territorio y lenguaje e interactúan interiorizando los procesos sociales, ellos construyen significados en el esquema de conciencia social, capacidades, intereses grupales, abordadas por sus propias 
experiencias, trayectorias y personalidades. Esto significa que cuando una comunidad cuando se ubica en fronteras, tiene sus propias diferencias culturales, son localidades territoriales con enfoque multicultural y heterogeneidad, que les permiten hacer uso de éstas como estrategias competitivas. Las fronteras territoriales tienen su interés para crear sus identidades, desde ellas se comienza a formar la noción de pertenencia a un país o territorio (Flores $\mathrm{H}, 2005)$.

En efecto, el propósito general de este trabajo se conjuga en la descripción de la identidad cultural desde la óptica de la arquigrafía, sobre las ciudades de fronteras a través de su esencia misma, como estrategia competitiva del turismo en el desarrollo económico local. Es decir, se asume el compromiso de base estratégica competitiva en el desarrollo económico local, para territorios ubicados como ciudades de fronteras desde la óptica de una promoción turística de la identidad, juegan un papel esencial los valores potenciales de comunicación visual empresarial, donde la arquigrafía y la arquitectura gráfica, son campos que permiten proyectar espacios geográficos en una localidad.

Una de las alternativas que presentan las ciudades de frontera para hacer de su visión un potencial, es su propia identidad cultural en función de la competitiva estratégica local. Se observa como cualquier grupo social, emana desde sus enfoques cotidianos sus teorías de la identidad y cultura en el entorno,, dadas las circunstancias presentes, hacen que todo ciudadano tome conciencia de su cultura, ya es una certeza que en ellos se experimenten su distintividad en la identibilidad social y singular, pues, es evaluada en la praxis frecuente respecto al análisis de la 
sociedad, siempre relacionadas con el desarrollo económico local (Flores H, 2005).

La estructura de este trabajo es la siguiente: en primer lugar, una aproximación al concepto la identidad cultural como atribución elemental de ciudades de fronteras, con un pasaje por a) las teorías antropológicas desde la perspectiva de la identidad cultural y b) las ciudades y comunidades sostenible en el ámbito de la identidad cultural, y c) la promoción de la identidad cultural y arquigrafia para un potenciado económico en las ciudades de fronteras. En segundo lugar, y teniendo en cuenta el objetivo de identidad cultural se analiza la estrategia competitiva como visión modal viable de ciudades de fronteras, una modalidad potencial de localidades para su desarrollo. En tercer término, se describirá el Desarrollo económico local de las ciudades de frontera. Por último, se recoge algunas reflexiones y visión sobre los objetivos del desarrollo del milenio.

\section{La identidad cultural como atribución elemental de ciudades de fronteras}

La identidad cultural de las ciudades de fronteras, prevalecen en los grupos sociales, que remiten fundamentalmente al estudio de contextos y circunstancias desarrolladas en el ámbito de la cotidianidad. Allí nace la complejidad cultural con perspectiva a las representaciones sociales, es decir, una cultura local vale desde el proceso de reivindicación de la distintividad, y puede sobrevivir ante las incursiones de los procesos de globalización que penetran desde el exterior. Entendiendo que es un proceso dinámico el desarrollo de 
las sociedades, y que la estabilidad y el cambio, funcionan desde el interior de todo sistema y los filtros culturales (Flores H, 2005).

La identidad cultural es un proceso de diferenciación de carácter intersubjetivo. ¿Qué significa esto? obviamente construye identidades de diferenciación. Vinculando esta concepción con la postura de Habermas, la identidad cultural es tradición, es construcción social e histórica, es pasado, presente y futuro; pero aspecto un interesante, se constituye a razón de varios aspectos o niveles de esencia de una localidad (ver gráfico n.1).

Estos son niveles de esencia del ser nacional bajo un desarrollo de autonomía y dinámica endógena, que todo habitante de una localidad fronteriza defiende por su ética y costumbres ancestrales, prevalecidos a su historia (Vergara Estévez \& Vergara D., 2002).

\section{Teorías antropológicas desde la perspectiva de la identidad cultural}

Las teorías de la identidad cultural, en especial cuando se tratan de las ciudades de frontera, en ella la situación sobre las perspectivas de la economía mundial. En ese particular, se explana que el deterioro de identidad, esta visto desde múltiples aspectos de las perspectivas económicas mundiales, la cual, puede complicar la consecución de los objetivos en desarrollo, especialmente en las ciudades que confrontan las zonas fronterizas, ellas potencian sus identidades culturales como pilar fundamental de las economías (ONU, 2019). 


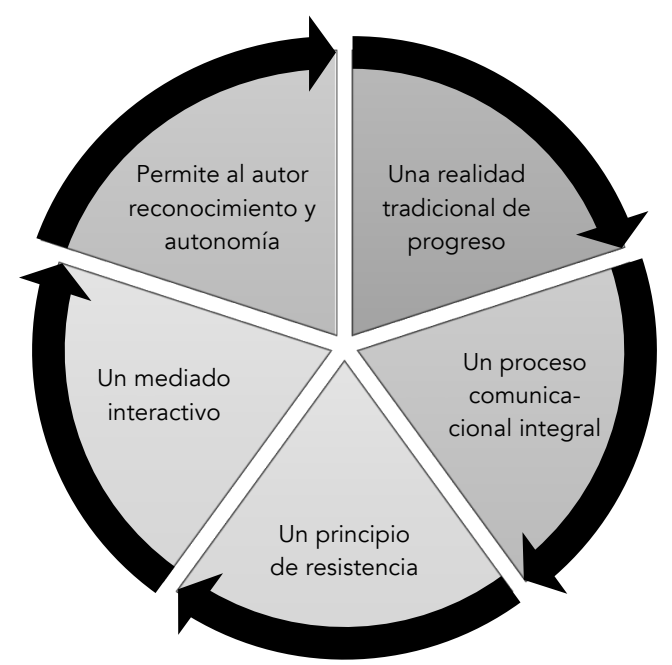

La identidad cultural como sentimiento de pertenencia de un espacio social; son vertientes importantes, que se conjugan como un todo, en una discusión teórica. ¿Qué sucede con estos términos? Todo ello, se atomiza en las llamadas teorías antropológicas, subsumidas desde las estructuras y sistemas, donde las personas en sus praxis sociales que forman parte de los procesos de cambio en las mismas comunidades (Flores H, 2005).

Así las cosas, la identidad local, es pertenencia, sentida de distintividad espacial y social, cada individuo toma conciencia de su cultura y cada organización social posee sus propios elementos sustanciales representativos en el campo de las identidades, como estructura de la vida social local.

Pero, se debe asumir lo vulnerable que es al centralismo del Estado. Los procesos de conformación de la identidad y sus formas de expresión cotidianas son dinámicas e interactivas, no así los procesos de cambio, pues, las comunidades se perciben y representan por sus individuos, en pequeñas comunidades, y datan de las teorías 
antropológicas contemporáneas con incidencia de la globalización respecto a lo cultural. Esta última da lugar a las sociedades tecnificadas con identidad, llena de valores hacia las zonas periféricas.

Asumiendo las teorías antropológicas, estas se vinculan con las vigentes teorías de Porter (explicadas más adelante en estrategias), que en razón a su contexto globalizador existen las teorías empresariales más recientes generadas en el libro The Discipline of Market Leaders (La disciplina de los líderes del mercado), cuyos estudios son de Michael Treacy y Fred Wiersema (Aste, s/f). Ellos desarrollaron las claves de una estrategia competitiva en base a tres disciplinas de valores, estas disciplinas subsumidas empresarialmente constituyen a base de la excelencia operativa, el liderazgo del producto y la intimidad del cliente.

Gráfico nro. 2. Las claves de una estrategia competitiva

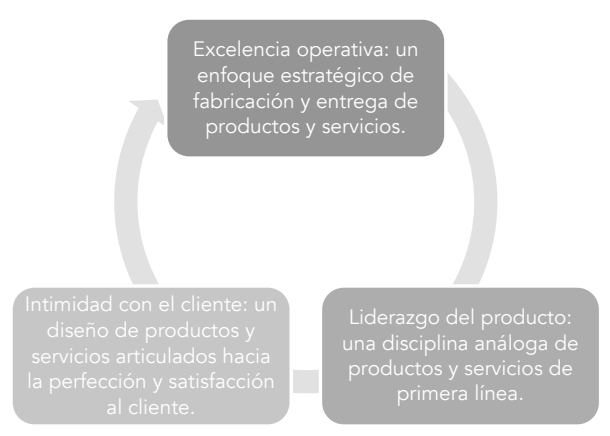

Otras de las clasificaciones existentes sobre las teorías de la cultura nos encontramos con las teorías materialistas como sistemas adaptativos, y las teorías ideacionales. Entonces, cada una de ellas tiene sus acepciones propias.

En primer lugar, las teorías materialistas de la cultura como sistemas adaptativos, son soportadas en el entretejido del diseño de los componentes biológicos y culturales del humano mediante el aprendizaje 
cultural9 . También las coincidencias de los representantes de las teorías de sistemas adaptativos, son las siguientes: a) Las culturas como sistemas relacionan comunidades humanas entre entornos ecológicos; b) El cambio cultural como proceso de adaptación al medio y ámbito natural, c) Los aspectos culturales que conforman los procesos de cambio social son la tecnología, la economía de subsistencia y los elementos de la organización social directamente ligados a la producción, y d) Los componentes ideacionales de los sistemas culturales pueden tener consecuencias adaptativas (Enguix, 2010).

En segundo lugar, las teorías ideacionales de la cultura, se pueden describir a través de la presentación de sus tres enfoques en que se entreteje, son: a) Las culturas como sistemas cognitivos: contempla un sistema de conocimiento, bajo un código de lenguaje organizado como sistema cognitivo general, b) Las culturas como sistemas estructurales: contempla las culturas como sistemas simbólicos compartidos que son acumulativas creaciones de la mente, estructurados desde los dominios culturales, y c) Las culturas como sistemas simbólicos: contempla las culturas como sistemas de símbolos y de significados compartidos (Enguix, 2010).

\section{Ciudades y comunidades sostenible en el ámbito de la identidad} cultural

Las ciudades de frontera son comunidades sostenibles en una localidad o ciudad cercana a la frontera, pudiendo estar entre dos 
países, estados o departamentos, o regiones o municipios. Normalmente el término implica que la cercanía a la frontera es uno de los aspectos por los que el lugar es más conocido según sus costumbres y cultura participativa. Debido a su proximidad a un país diferente, puede tener influencia de tradiciones y cultura exóticas en el lugar. Las ciudades de fronteras siempre han sido los motores de procesos y cambios de una sociedad, aunado a su innovación social constante, proporcionando espacios inspirados en su desarrollo económico local aceptable en la competitividad nacional (TINA Viena, Departamento Municipal 18, 2016).

Las ciudades fronterizas pueden tener comunidades altamente cosmopolitas, una característica que comparten con ciudades portuarias, debido a los constantes viajes y comercio que pasan por la ciudad. También pueden ser puntos en que se generen conflictos internacionales, especialmente cuando los dos países tienen disputas territoriales. Pero, a fin de cuentas, se generan en ellas las comunidades sostenibles, es decir, aquellas que, por sus capacidades comerciales y económicas, generan desde lo particular a lo público, condiciones de temporalidad indefinida, capaces de mantenerse en el tiempo y generar incluso dar apoyos a sus coterráneos o vecinos ${ }^{10}$.

En medio de prolongadas disputas comerciales y de una gran incertidumbre política, en el último año diversos aspectos de la economía mundial se han deteriorado considerablemente, lo cual podría obrar en contra de los esfuerzos por reducir la pobreza, crear empleos decentes, ampliar el acceso a la energía asequible y limpia y alcanzar muchos otros

\footnotetext{
10 Los ambiciosos objetivos de la Agenda 2030, cuya visión está dada por el Desarrollo Sostenible, la economía mundial tiene que ser dinámica e inclusiva.
} 
Objetivos de Desarrollo Sostenible (Agenda para el Desarrollo Sostenible, 2020).

En este propósito, la serie de cifras y estadísticas, y el crecimiento del producto mundial bruto, la cual se redujo al 2,3\% en 2019, el nivel más bajo desde la crisis financiera mundial de 2008-2009. Esta desaceleración se produce al tiempo que se acentúa el descontento con la calidad del crecimiento económico desde el punto de vista social y ambiental, en un contexto de desigualdades generalizadas y una crisis climática creciente, subsumidas en los temas de la heterogeneidad y multiculturalismo, como pilares del desarrollo sostenible (ONU, 2019).

Aunque las tensiones comerciales mundiales están disminuyendo en algunos frentes, el riesgo de recaída es alto, puesto que estas disputas responden a problemas importantes que todavía no se han abordado con detenimiento; siempre que algunos posibles reveses no lleguen a producirse. Ya, para el año 2020, se prevé un modesto aumento del crecimiento mundial, que se cifraría en el 2,5\%, aunque la incertidumbre en materia de políticas seguirá afectando a los planes de inversión. ¿Qué significa esto? Para las ciudades de frontera, el desarrollo económico local, se les hace un abismo, pues, no son sus fortalezas las que se mantendrían como bases estratégicas competitivas, no así, la identidad cultural, en cuyo caso sería el camino a tomar para resurgimiento económico poblacional local.

Cuando se trata el tema de la heterogeneidad y multiculturalismo, son producidos desde la racialización de las personas, que no es otra 
cosa, identidades en términos de raza, con óptica de las ciencias sociales y humanas (Rodríguez, 2000).

Se infiere el contexto sobre las identidades en términos de raza, pues, ambas la heterogeneidad y multiculturalismo, coinciden en el grado de incidencia, la primera como cultura desde el pasado para recuperar datos de casuística en reconstituir las genealogías del conocimiento respecto a la política, y la segunda es política pública de una sociedad. Se aprovechan ambas como estrategias para localizar a los pobladores dentro de las fronteras del Estado.

Definitivamente, una localidad, presenta sentimientos diferenciales entre sí, lo que podría denominarse conciencia cultural, y reconoce límites y fronteras, sus comportamientos no pertenecientes a la localidad no conocen normas ni valores, pues, son temporales, y son los no nativos. Pues, existen tradiciones que poseen los locales con una vida social comunitaria. En ella se conjuga la tradición y la costumbre, ambas en una sociedad periférica, posee sus tradiciones, valores y normas, y se caracterizan por su permanencia o invariabilidad, la costumbre o ley común de variabilidad y adaptabilidad, se arraigan de la tradición como valor pragmático de una ciudad de frontera con identidad, atomizadas por las denominaciones de heterogeneidad y multiculturalismo social, que harán que una ciudad de frontera prospere en función al desarrollo económico local, producto de su identidad cultural.

Hechas las consideraciones anteriores, se puede resumir que las metas que persigue el Objetivo 11 de la Agenda 2030, trata las materias previstas en la tabla 1. 
Esto significa, que de alguna manera los factores externos e internos incidan en el desarrollo sostenible de las ciudades locales, pues, se visionan a nivel mundial aspectos donde los organismos internacionales consideran ser de relevancia para los pobladores, incluso hace énfasis en aquellos cuyas situaciones de vulnerabilidad les afectan, y por ende entran a jugar papel preponderante las ciudades fronterizas por su fragilidad en términos de políticas y cultura, por eso se deben fortalecer en la sostenibilidad y sustentabilidad.

Tabla 12. Objetivo 11 de la Agenda 2030. Acciones y materias que seguir

\begin{tabular}{|c|c|}
\hline Acción & Materia y término que persigue \\
\hline \multirow[t]{2}{*}{ Asistencia } & Personas en situación de vulnerabilidad \\
\hline & Financiera a países necesitados \\
\hline \multirow[t]{2}{*}{ Construcción } & Ciudades, urbanismos, viviendas y asentamientos humanos con servicios básicos \\
\hline & Inmuebles, sostenibles y resilientes utilizando materiales locales \\
\hline Control & Pérdidas económicas según producto interno bruto mundial \\
\hline \multirow[t]{2}{*}{ Creación } & Sistemas de transporte y vialidades \\
\hline & Zonas verdes y espacios públicos seguros \\
\hline Gestión & Patrimonio cultural y natural del mundo \\
\hline Integralidad & Riesgos de desastres a todos los niveles \\
\hline Mitigación & Cambio climático, impacto ambiental, calidad del aire y la gestión de los desechos \\
\hline Planificación & Desarrollo y optimización de recursos \\
\hline Políticas & Planes integrados subsumidos en lo económico, social y ambiental \\
\hline Reducción & Riesgo de desastres, muertes y personas afectadas y resiliencia \\
\hline
\end{tabular}

Promoción de la identidad cultural y arquigrafia

El tema de la promoción de la identidad cultural la iniciamos con una inquietud, y es que la Arquigrafia juega papel preponderante en las ciudades de frontera para lograr un potenciado económico, entonces ¿Cómo se promociona la identidad cultural para un desarrollo económico sostenible? Previstas las condiciones sobre la identidad cultural, se puede inferir que se debe considerar en primer 
término el sentimiento de pertenencia, y luego los accesorios términos de promoción que forman parte de un grupo social tales como los valores, principio, orgullos, costumbres, creencias, comportamientos, entre otros, desde el enfoque de la promoción cultural como herramienta para fortalecer la identidad (Ballesteros, Gracia, Ocaña, \& Jácome, 2018).

Se infiere la postura de los autores, pues se podría resumir sus intenciones en cuanto a los aspectos que deben considerarse para promocionar la identidad cultural y tradicional como fuente de diferenciación, con sentido de empoderamiento de una ciudad de fronteras, tomando en cuenta que la globalización y la desculturización como proceso, inciden y serían en todo caso de gran relevancia para quienes se avocan a la realización de promociones, a través de campañas de difusión, se aprecian los aspectos en la tabla 2.

Tabla 13. Aspectos que deben considerarse desde la arquigrafia para promocionar la identidad cultural de una ciudad de fronteras

\begin{tabular}{cc}
\hline Aspecto a considerar & Actividades de Identidad cultural como fuente de diferenciación \\
\hline Costumbres & Ancestrales y diversidad \\
Creatividad & Conducción de procesos culturales \\
Proceso & Cultural en gestión, administración, diferenciación e innovación \\
Festivales & Culturales consecutivos \\
\hline Instituciones educativas & Rescate, preservación y protección cultural \\
Manejo asertivo & Cultura y potenciación local y externa \\
Necesidad & Consumidores culturales \\
Valores & Culturales y transculturización \\
Visiones nuevas & Estrategias laborales con capacidades y negocios \\
Actividades & Lúdicas o recreativas \\
\hline
\end{tabular}


Las localidades territoriales y su sentido de pertinencia de una región, puede proyectarse a través de la arquigrafía, sus actividades culturales, entran a formar parte de un proceso para lograr hacer de ésta una marca identificadora. La razón sobre este temario es que parte de un sistema gráfico, es decir, pone al público en contacto sobre un determinado lugar, ¿para qué se hace? Se hace para indicar: dónde está, cómo moverse y hacia dónde. Estos aspectos hacen que los visitantes de una ciudad determinada, en especial las fronterizas, gocen al sentir que se les ofrece seguridad, recorrido, confort, estancia y sobre todo la imagen cultural que destaque el símbolo territorial en función a la arquigrafía dada.

En ese orden de ideas, la arquigrafía presenta desde la óptica comunicacional una herramienta de transmisión de mensajes, tan potente que puede darse en la representación hacia las comunidades con perspectiva de aplicabilidad arquitectónica, cuya razón gira en un gran abanico de ventajas, para atraer a la población y su admiración por el mensaje que pueda transmitir. Siendo un potencial ante la competencia, cuando se relaciona marca, espacio y producto, pues la arquigrafía debe considerar sus tres dimensiones, es decir, que una ciudad fronteriza, puede ser abordada con estos elementos para ayudar y orientar al usuario a conocer la potencialidad que puede ofrecer una ciudad de frontera, su desarrollo económico local.

La competencia existente entre las ciudades por captar la atención y posicionarse como mejor destino turístico se manifiesta en forma de potentes marcas que compiten entre ellas al igual que los productos de cualquier lineal. El nombre de una ciudad es en sí mismo una marca, 
pues la población relaciona un nombre a unos valores culturales, sociales y económicos, con incidencias de la globalización, es decir, que una buena marca ciudad favorecerá la proyección internacional de esta en términos de potencialidad.

Entonces es evidente que, el tema de la globalización es uno de los aspectos componentes del sistema de la promoción de la cultura en ciudades de frontera, y sobre el particular, y en este contexto fronterizo esta la dimensión cultural y económica, pues, busca espacios comunes de desarrollo, la cual las líneas demarcatorias hacen ruido a las culturas y formas de vida asociadas, siempre relacionadas con la cooperación e integración. Las ciudades de fronteras son pueblos llamados a profundizar sus identidades culturales que desde la globalización tiene su propia incidencia e impacto (Ver gráfico nro.3).

Teniendo en cuenta que la globalización tiene su incidencia en la promoción de la identidad de la cultura en localidades ubicadas en fronteras, sus acepciones se ver reflejadas en el gráfico n.3. Dichas acepciones recogen el tema de expansión humana, fenómeno natural, marco referencial, acción organizacional internacional, crecimiento productivo, y la promoción cultural. Todas se conjugan en los episodios por las cuales es sometida la identidad cultural de una población en fronteras (Sociedad Geográfica de Colombia, 2006).

En resumidas cuentas, una marca ciudad, bien construida y coherente, ayudará a desarrollar una narrativa consistente, independientemente si es o no incidida por la globalización, destacando los aspectos que la hace singular e inimitable. Será desde luego un potenciado económico sobre una razón fáctica, al tema referido sobre ciudades de fronteras cuya 
existencia viven pobladores que se diferencian en algo nuevo como patrones de conducta cultural, es decir, una marca global que genere proyección mundial por su relevancia, pudiendo ser o no potente, pues un área o zona de la ciudad de frontera, consigue acercarse a ser turística, comercial, atractiva estudiantil, o de negocios; representando una estrategia competitiva del desarrollo económico local.

Gráfico nro. 3. Acepciones en la globalización para la promoción de la identidad cultural.

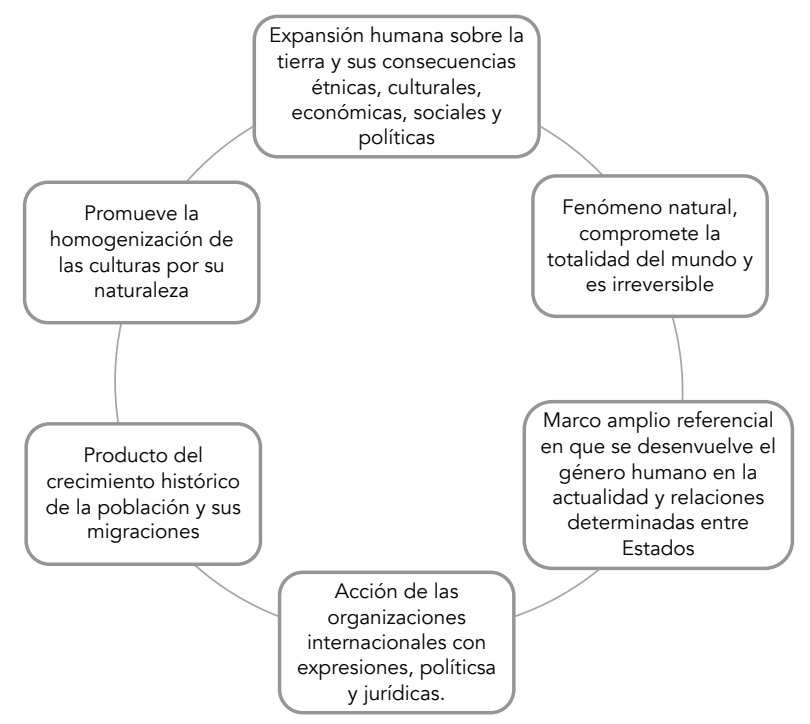

Estrategia competitiva como visión modal viable de ciudades de fronteras

El concepto de estrategia competitiva viene dado de acuerdo a la teoría más reciente de Michael Treacy y Fred Wiersema, por una complejidad asumida de varios autores, entre ellos Banco Europeo de Inversiones (2015), Schwab (2014), Turok (2004), todos ellos coinciden que, son planificaciones, realizadas en función a las fortalezas y habilidades de aquellas empresas motoras, en función a movilizar y utilizar eficaz y eficientemente los recursos productivos necesarios y a 
su alcance, en el contexto de ofrecer bienes y servicios productivos y generadores inmersos en las políticas económicas globalizadas (García Nicolás, 2015).

De la misma manera, la importancia dada a las estrategias competitivas se asume desde el enfoque de la productividad-país. Esto significa que, la competitividad como modalidad potencial de una localidad X, es determinante en función a tres elementos subsumidos en ello, y son: el incremento, la recuperación y la inversión, es decir, los tres elementos son los representantes de la garantía potencial de un crecimiento económico para determina ciudad territorial.

La competitividad se debe asumir entre los elementos sustanciales, los pilares sobre los que se asienta ésta, y no es otra que la misma innovación orientada hacia el crecimiento inteligente, sostenible e integrador que toda localidad territorial deberá connotar en sus políticas públicas (García Nicolás, 2015)

El tema de la competitividad estratégica, ha sido desarrollada en Europa y se ha asumido con tendencia hacia América Latina para el 2020, estos aspectos consecuentes son de relevancia económica para las ciudades de frontera, y son los que están fusionados con los mercados competitivos y flexibles, tomando como primer término el sector financiero de inversión, y en segundo lugar, las infraestructuras estratégicas referidas a transporte, energía y medio ambiente, sin dejar uno de los pilares esenciales que es el capital humano, su formación y salud (García Nicolás, 2015).

En este recorrido, encontramos los objetivos que persigue la Estrategia Europea durante 2020 y siguientes, estos son la globalización 
y las transformaciones sociales, que bien son parte consecuente de América Latina, incluida la solidaridad y la justicia social como principios fundamentales.

En las mismas circunstancias y respecto a las estratégicas competitivas enmarcadas por la modalidad potencial, territorialmente ubicadas en las ciudades de fronteras, se desprenden diferentes concepciones y autores, entre ellas las referidas en la tabla 3.

Tabla 14. La competitividad y sus concepciones según autores

\begin{tabular}{|c|c|c|c|}
\hline Autor & Año & Competitividad & Concepción \\
\hline $\begin{array}{l}\text { García } \\
\text { Nicolás }\end{array}$ & 2015 & Regional & $\begin{array}{c}\text { Capacidad de una región para generar ingresos y mejorar la calidad de } \\
\text { vida de sus habitantes, sin vincularla como empresas o versión a escala } \\
\text { país. }\end{array}$ \\
\hline $\begin{array}{l}\text { Huggins y } \\
\text { Thompson }\end{array}$ & 2012 & Empresarial & $\begin{array}{c}\text { Nivel del capital humano, el grado de capacidad innovadora y la } \\
\text { calidad de la infraestructura local. }\end{array}$ \\
\hline $\begin{array}{l}\text { Huggins y } \\
\text { Clifton }\end{array}$ & 2011 & Económica & $\begin{array}{c}\text { Política de desarrollo económico con apoyo a la creatividad de } \\
\text { elementos interrelacionados. }\end{array}$ \\
\hline $\begin{array}{l}\text { Jansson, } \\
\text { Waxell }\end{array}$ & 2011 & $\begin{array}{l}\text { Desarrollo y } \\
\text { calidad }\end{array}$ & $\begin{array}{l}\text { Todos conjugados en la globalización, unifican la producción vinculada } \\
\qquad \text { a la calidad. }\end{array}$ \\
\hline Malecki & 2007 & Industrial & $\begin{array}{c}\text { Capacidad de las empresas con tejido basado en sectores específicos } \\
\text { con unas instituciones especializadas y con políticas de objetivos } \\
\text { definidos, y unas estructuras de gobernanza que faciliten la relación } \\
\text { entre el estado y los actores sociales. }\end{array}$ \\
\hline Meyer, E. & 2008 & Sistémica & Habilidad de una localidad para generar elevados y crecientes ingresos \\
\hline Gardiner & 2004 & & $\begin{array}{l}\text { y mejorar el nivel de vida de las personas que la habitan, hacen la } \\
\text { fórmula: Productividad + competitividad= prosperidad. }\end{array}$ \\
\hline Camagni & 2002 & Regional & $\begin{array}{l}\text { Capacidad de los territorios de proporcionar un entorno competitivo a } \\
\text { las empresas junto con procesos de acumulación de conocimientos }\end{array}$ \\
\hline
\end{tabular}

Como puede observarse en las diferentes concepciones según sus

autores Huggins y Thompson (2012), Huggins y Clifton (2011), Jansson

Waxell (2011), Malecki (2007), Meyer (2008), Gardiner(2004), Camagni

(2002) y el propio García Nicolás (2015), se puede inferir que la Competitividad, la rige como norma rectora la Capacidad, Habilidad - Política, la cual abarca regiones, territorios, localidades, instituciones, actores sociales, pero siempre en la búsqueda de la mejor vida de los ciudadanos vinculados a la calidad tanto del Estado, Comunidad y Empresas. 
Otra de las consideraciones habidas en la tabla 3, es novedosa para seguir las estrategias de potencial de las ciudades de fronteras, refiriéndose a que la clasificación viene dada desde el Desarrollo y calidad, Económica, Empresarial, Industrial, Regional y Sistémica. Se infiere que cualquiera que fuese el tipo de Competitividad, siempre buscará los logros de productividad y prosperidad, en función a la capacidad para optimizar el desarrollo económico existente de una localidad o región. Sobre la base de las concepciones, se encuentra la fórmula del Diamante de Porter (Flores Hernández, 2013).

Para dilucidar detalladamente los factores vinculados a la competitividad y como lograr el éxito, son aspectos explanado en el gráfico 4.

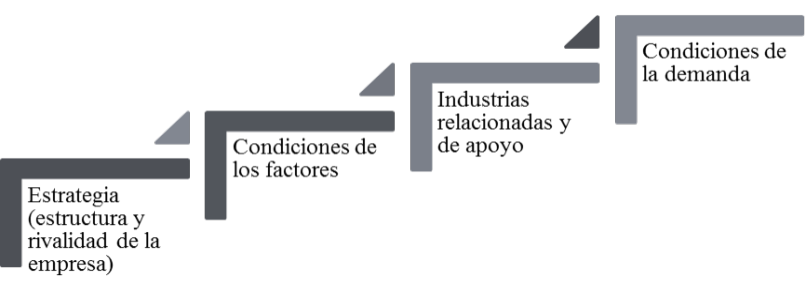

Gráfico nro. 4. Las cuatro claves que se representan el diamante de Porter para considerar una región competitiva

Cualquier región se puede identificar con los cuatro aspectos claves que se representan en el diamante de Porter. ¿Qué sucede con el diamante de la competitividad? En él se aprecian desde el contexto del desarrollo económico, industrial y la propia competitiva: el elemento 
Estrategia (estructura y rivalidad de la empresa); las Condiciones de factores; las Industrias relacionadas y de apoyo; y las Condiciones de la demanda. Además, se asume que, el Gobierno y la casualidad (azar) son factores que determinan las ventajas competitivas de una localidad, es decir, que nos permite visualizar la estratégica competitiva según la modalidad potencial de una localidad, o en este temario a una ciudad de frontera. ¿Qué quiere decir esto? A simple vista se resume los objetivos que persigue la competitividad (Flores Hernández, 2013). A continuación, están representados en el gráfico 5.

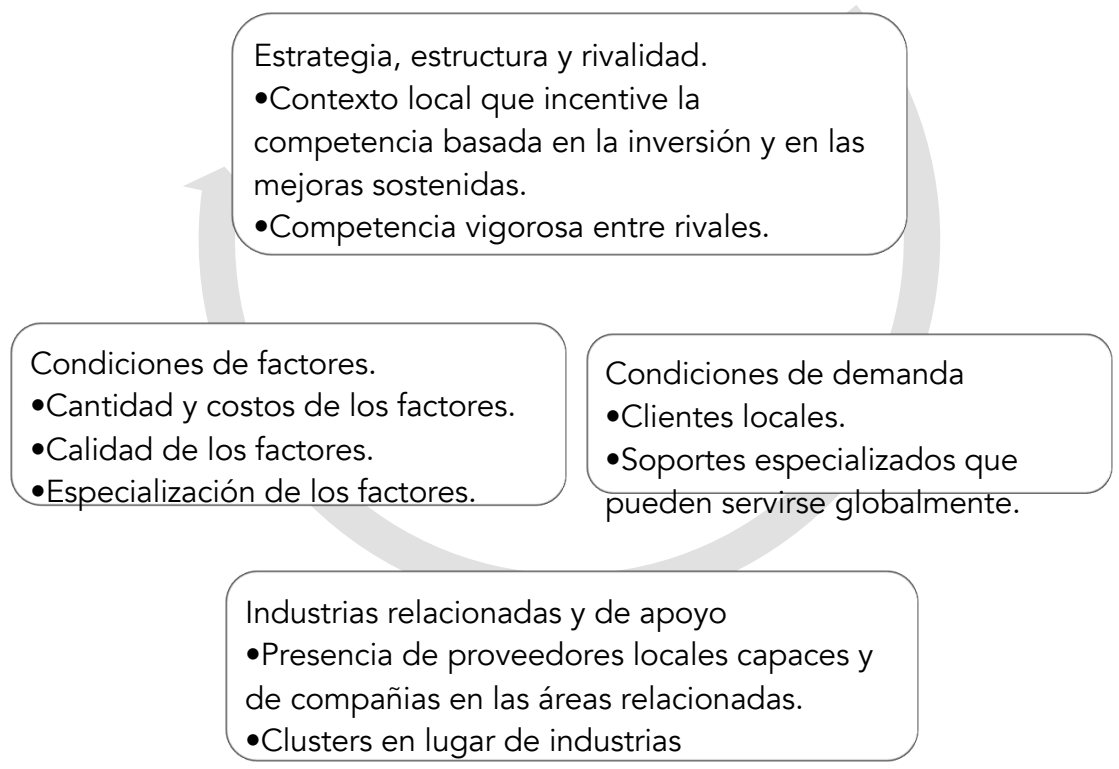

Gráfico nro. 5. Factores vinculados a la competitividad y como lograr el éxito según el Diamante de Porter.

Como puede observarse, el diamante de Porter ejemplariza el contexto de la competitividad, y se reflexiona a que el sistema de actividades económicas, incide en la integración generalizada hacia un valor e impacta en su productividad (Flores Hernández, 2013). 
En otras palabras, es el sistema de proceso productivo, que permite alcanzar la ventaja competitiva de las empresas cuando son capaces de aprender rápidamente.

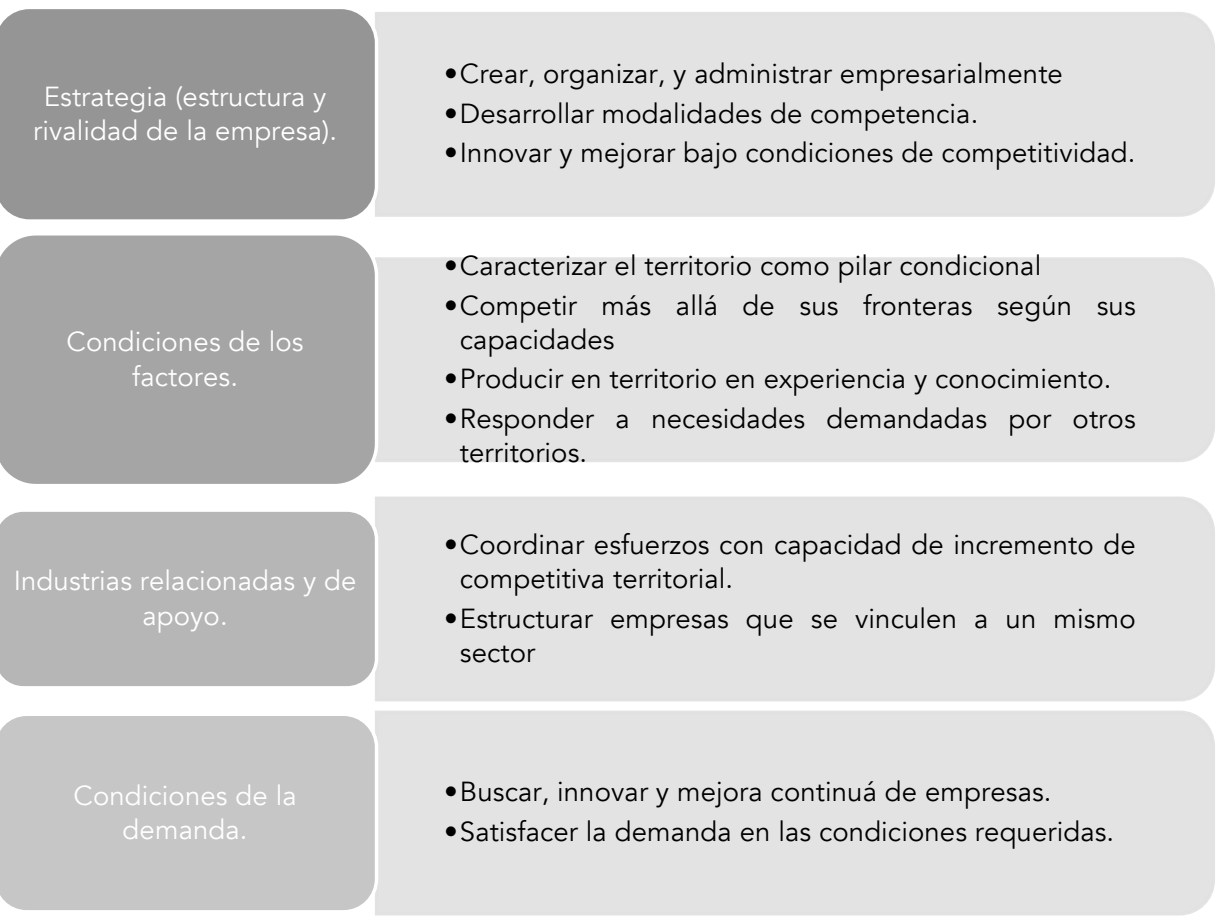

Gráfico n. 6. Descripción de los factores de competitividad de Porter, tomado de Flores Hernández (2013) adaptado por el autor

Para llegar a la competitividad y estimulación de empresas según este gráfico, se deben considerar los factores de competitividad, y abordarlos en función a un mejor rendimiento, apoyados de las mejores técnicas y la acumulación de activos existentes, según las políticas públicas que el Estado facilite y genere en la gestión empresarial. Pero, no obstante, se debe asumir que la competitividad en los mercados es la que, determinada la producción, según la capacidad de los actores que deberían influir positivamente en su entorno o localidad para conseguir el éxito. 
Desarrollo económico local de las ciudades de frontera

El protagonismo de actores locales en la formulación de estrategias se conjuga bajo la visión del desarrollo económico local dentro de un proceso nacional, fundamentado en la promoción de líneas estratégicas que permitan un desarrollo y progreso de una localidad. Como baluarte de este proceso, se haya el tema de la toma de decisiones, pues, en ella se puede implementar nuevos proyectos, programas y planes, que satisfagan como iniciativas novedosas a la cooperación cultural fronteriza. Existen cuatro importantes líneas de Cooperación cultural transfronteriza que Fronteras Abiertas asumen dentro del proceso de construcción social, a través de la cultura y el desarrollo económico local de ciudades de frontera (Rhi-Sausi \& Conato, 2009). Ver gráfico 7.

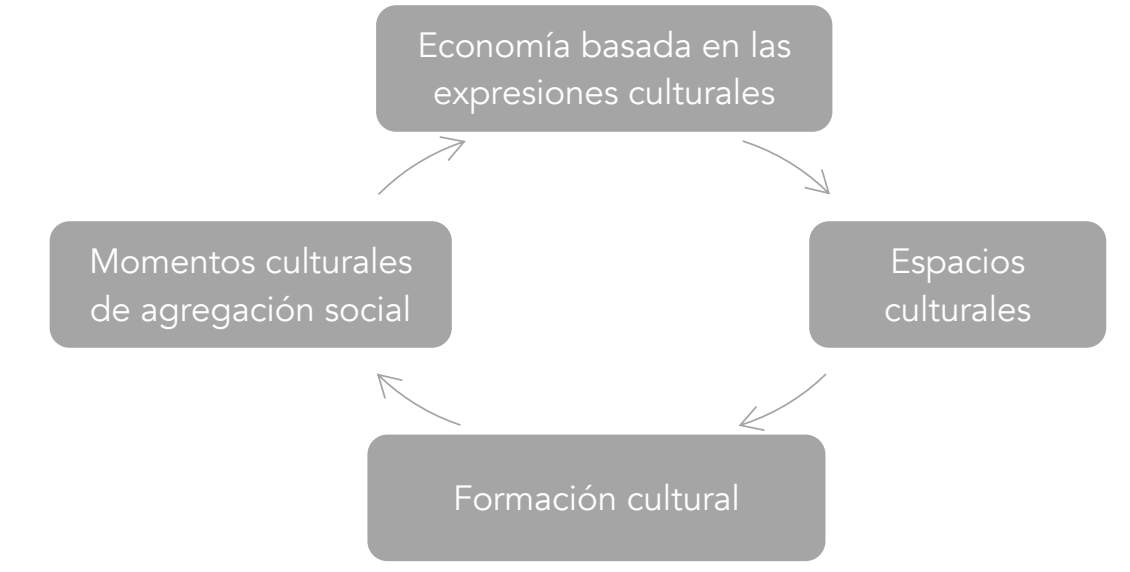

Gráfico nro. 7. Líneas de cooperación promocional de la cultura transfronteriza. Fronteras abiertas (RhiSausi y Conato, 2009)

Como puede observarse en el gráfico 4, las líneas de cooperación cultural transfronteriza, se estiman en cuatro vertientes para su promoción, entre ellas nos referimos así: a) Espacios culturales. En ella 
se reseña la infraestructura física, esta permite desarrollar las actividades que satisfacen y dan continuidad a la demanda cultural real, según su potencial, con visión a la integración y creación de espacios culturales. Hay entonces fronteras secas, en cuyo seno se hace necesario acudir a la creación de espacios culturales en la línea fronteriza; b) Formación cultural. Da lugar a programas de cooperación cultural transfronteriza, con prioridad al desarrollo, producto a la demanda de formación, pues debe proporcionar recursos disponibles, c) Momentos culturales de agregación social. Las festividades populares anuales, son la principal forma de expresión de la identidad cultural de las ciudades fronterizas. Su propósito es involucrar a la comunidad vecina a temas identitarios que estén definidos en el enriquecimiento y diversificación de la oferta cultural; d) Economía basada en las expresiones culturales. La creación de economía cultural permite que se disponga de un desarrollo local, con cooperación cultural transfronteriza, pues, en ella se propone proyectos de desarrollo económico local.

En ese orden de ideas, se puede apreciar que el proceso de desarrollo económico local versa sobre los aspectos de autonomía y dinamismo, ambos actúan frente a las agentes locales embargadas por la situación social que actualmente viven los pobladores, no es otra que la situaciónpaís. Es un reto impulsar el desarrollo económico local y obtener una mejor situación social y económica en el ámbito de las tendencias generales de las políticas generadas por el Estado para las ciudades de frontera.

En el V Foro Mundial de Desarrollo Económico Local , Ilevado a cabo en Córdoba (Argentina), del 6 al 8 de mayo de 2020, dejó entrever la 
transformacion en alianza global para estimular las políticas de desarrollo local sostenible, su proposito fue erradicar pobreza, empleo, trabajo, espíritu empresarial sostenible, asociaciones de múltiples de participación de la sociedad civil, trabajadores y empleadores. Ademas, hace enfasis en el enfoque operativo y estratégico para localizar la Agenda 2030, contemplada en el Desarrollo Sostenible y sus objetivos. Colombia fue sede del Tercer Foro Regional de Desarrollo Económico Local para América Latina y el Caribe realizado en Barranquilla, Colombia en 2019 (Observatorio de Desarrollo Local, 2020).

En el tema de las estrategias de desarrollo económico, se puede expresar que forma parte de ello, el desarrollo local, pues, desde la misma administración pública, cumple el papel interpretativo de las pequeñas y medianas empresas en la región. Por otro lado, el cambio de la situación y el rol de la administración sobre las nuevas empresas deben beneficiarse en el ámbito geográfico como económico, siempre favorable a sus intereses económicos, donde los empresarios seleccionan la mejor implantación en función a su tejido empresarial y los servicios existentes, ejes de transportes, evolución industrial, tecnología, social y de adecuación a la producción de los mercados existentes, ente otros (Del consultores, desarrollo económico, planificación estrategica y emprendimiento, s/f).

Sobre el particular, los municipios evaluarían si le es o no favorables la implantación o renovación de empresas, si se puede o no incentivar el desarrollo de algún tipo de red productiva que generen ocupación estable, o si es viable otro tipo de desarrollo alternativo en la localidad, 
que permita tener una base estratégica de competitividad en el desarrollo económico local, en especial las ciudades fronterizas. Lógicamente, no existe un modelo de desarrollo local ideal, ni existe una estrategia común con garantía de éxito (Bárcena, 2019).

Esto se puede circundar cuando en el informe de la Comisión Económica Para América Latina y el Caribe, menciona que durante el periodo 2019, la economía del sector cerró con macro crecimiento de 0,1 por ciento, aunque en 2014, fue peor lo cual refleja en conjunto la peor actuación de la región en los últimos años. Es decir, se proyecta un crecimiento de 1,3 por ciento para la región en 2020 y siguientes de forma sistemática, y sin control con una desaceleración en la demanda interna, una baja demanda agregada externa y mercados financieros internacionales más frágiles; novedosas crecientes demandas sociales, reducción desigualdad y aumento a la inclusión social (Bárcena, 2019).

Se observa claramente, como esta situación nos cobija en el marco de las políticas activas de ocupación, es decir, la inserción laboral o el mantenimiento de la ocupación, con bienestar sobre cinco aspectos que deben considerarse como estrategias de desarrollo económico de cualquier localidad referida a frontera (ver gráfico 8).

El informe de la Comisión Económica Para América Latina y el Caribe, expresa con claridad las medidas tomadas y clasificadas en políticas sobre la orientación -relación oferta/demanda de trabajo-; la formación -mejorar conocimientos recursos humanos-; la promoción y creación de ocupación -nuevas ocupaciones-. El desarrollo local, visto desde este panorama, nos muestra la diversidad, los recursos que se pueden encontrar en una región, bajo los enfoques dinamizadores de actividad económica, centros 
productivos, logísticos, entre otros, a razón de las características del tejido empresarial.

Gráfico n. 8. Políticas activas en lo laboral de una localidad fronteriza

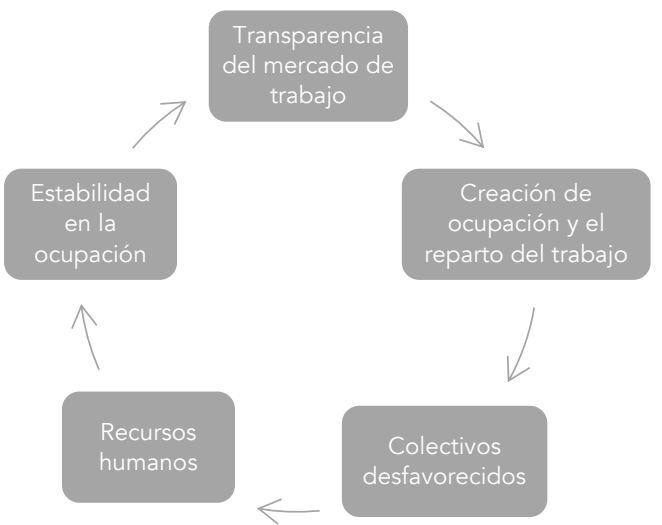

En ello, encontramos que los procesos de naturaleza endógena en ciudades de fronteras, como su desarrollo económico local, orbitan en la solidaridad territorial e identificación entre actores, los recursos endógenos desde una perspectiva dinámica e integral; y los de estructuras flexibles. Pero, existen situaciones incómodas en materia de proyecciones de crecimiento de ciudades locales en frontera, es qué, su economía presenta y presentará una desaceleración económica con márgenes impresionantes de promedio de expansión.

Entonces, cuando se presentan los datos de proyecciones de la Comisión Económica para América Latina y el Caribe, para el año 2020 y sucesivos, se observan datos astronómicos con la venía del COVID19, un balance preliminar demuestra países como Venezuela, país frontero con Colombia, expone un retroceso bajo contracción económica por debajo del -25\% para finales del 2020 (DW.Español, 2020). 
Actualmente, en datos del informe de la Comisión Económica Para América Latina y el Caribe y la Organización Internacional del Trabajo, a razón del coronavirus, han mencionado que éste, avanza inexorable en América Latina, aproximadamente hace referencia a los siguientes datos:

Tabla 15. Cifras de la comisión económica para América Latina y el Caribe y la Organización Internacional del Trabajo sobre América Latina y del Caribe. Año 2020

\begin{tabular}{ccc} 
Inicios Coronavirus América Latina. & $\begin{array}{c}\text { Infectados } \\
640.000\end{array}$ & $\begin{array}{c}\text { Muertos } \\
35.000\end{array}$ \\
\hline Desarrollo del Coronavirus América Latina. & 5.000 .000 & Tendencia 270.000 \\
Empleo hoy en América Latina & Nuevos desempleos & (11,5 millones). Primer trimestre Año 2020. \\
Tendencia del empleo en América Latina & Desocupados & (37,7 millones). Cuarto trimestre Año 2020.
\end{tabular}

Estos datos, tienen un gran significado para las ciudades de frontera a nivel mundial, pues el coronavirus, ha perturbado con pronósticos astronómicos las cifras en materia económica y de empleo. Hubo en los últimos meses, una macro aceleración de contagios, por ejemplo, en América Latina y el Caribe, razón por la cual, es preocupante la afectación que ha ocasionado la pandemia (Del consultores, desarrollo económico, planificación estrategica y emprendimiento, s/f).

Respecto al párrafo anterior, se pregunta ¿Qué significa esto en términos de estrategia competitiva? Significa que las ciudades de frontera que no estén solidariamente sostenibles en materia de identidad cultural como base estratégica competitiva, no podrán sostenerse con un desarrollo económico local, cuya razón está dada producto de factores externos, tal es el caso de la pandemia. Se pierden puestos de trabajo, se cierran locales, restringen paso peatonal y vehicular, extremas medidas sanitarias y de seguridad, confinamiento de pobladores y aledaños, y una fuerza global de las políticas públicas en materia de salud y, la necesidad 
de que cada ciudadano tenga que educarse, recrearse, transitar libremente, entre otros.

En este orden de ideas se puede citar a la Organización Mundial de la Salud, la cual ha manifestado, a través de todos los portales desde enero de 2020, la declaración de Emergencia de Salud Pública Internacional, que conlleva un brote de enfermedad por un nuevo coronavirus en la provincia de Hubei, China (OMS, 2020).

Esta institución preocupada por la situación de envergadura indicó la existencia de un alto riesgo, por la enfermedad denominada coronavirus 2019 (COVID-19), se propaga como una pandemia mundial. En concordancia con la Organización Internacional del Trabajo, se puede entrever que el sector empresarial e industrial deben asumir responsabilidades para detener la propagación de la enfermedad, y cuidar las labores económicas que van en detrimento, pues, las autoridades sanitarias de todo el mundo se comprometen en emergencia, adoptar medidas extremas para contenerle, y así todos los sectores de la sociedad. Se infiere la situación planteada, la cual incide en el desarrollo económico local, afectando las economías de ciudades importantes, incluidas las de frontera, en el entendido que son localidades cercanas a la frontera entre dos países, por sus entornos departamentales, estadales, regionales o municipales.

Otras de la informaciones que preocupan en portales se encuentran por ejemplo el referido por -Coronavirus avanza en América Latina con sombrío impacto para sus economías donde reflejan una situación especial apegada a esta situación del pandemia con relación a los 
sistemas económicos, los cuales están siendo afectados directamente. (Publinews, 2020).

En este aspecto encontramos que Latinoamérica presenta cifras de afectados alrededor de 640 mil personas y dejado más de 35 mil muertos en la región. Cuando vinculamos esta situación en materia económica y de empleo, es preocupante en función al desarrollo económico local. Se insiste en los pronósticos, pues, los casos se aceleran mundialmente al multiplicarse los contagios en América Latina y el Caribe. Para ilustrar esto, en función a materia de desempleo y pobreza Publinews registró, que la pandemia puede llegar afectar las economías locales por desempleo, sin trabajo ni labores presenciales en empresas, en virtud de manejar cifras de fuentes oficiales que destacan los 5.000.0000 de infectados, por ejemplo, países como Brasil (311.000), Chile (60.000), Ecuador (35.000), Guatemala (2.500.000), México (7.000), Perú (109.000) (Publinews, 2020).

Todos estos países de alguna manera promueven el avance de la COVID-19 en Latinoamérica, y debido o producto de la crisis que se produce en el marco de polarizaciones políticas, impacta desde lo económico generalizado, confrontaciones gubernamentales, entre otras. ¿Qué significa esto en materia de desarrollo económico local? Para nosotros en Colombia, es una alerta, porque si no se toman las medidas necesarias de bioseguridad en el ámbito laboral, en el hogar, en las empresas e industrias, las consecuencias pueden ser aceleradas en los entornos de la economía y afectar directamente los empleos. Tanto la empresa privada como el Gobierno, estarán muy vigilantes de esta situación, para desacelerar los contagios y así volver a la normalidad. 
Reflexiones y visión sobre los objetivos del desarrollo del milenio

Los Objetivos de Desarrollo del Milenio de las Naciones Unidas, están enfocados en comprometer a los dirigentes mundiales a luchar contra la pobreza, el hambre, la enfermedad, el analfabetismo, la degradación del medio ambiente y la discriminación contra la mujer, entre otras (Organización Mundial de la Salud, 2020).

Toda esta tendencia, hace que la justificación del trabajo y su vinculación, estén en correspondencia con el objetivo referido al fomento de una asociación mundial para el desarrollo, pues, sobre países más débiles y con necesidades urgidas, es que debe ocuparse, entre ellos debe abordarse la necesidad apremiante en fronteras, pues, se destaca la importancia del aspecto salud, en cuanto a desarrollo progresivo realizado a la consecución de los Objetivos de Desarrollo del Milenio.

Se canaliza una serie de estrategias por medio del alcance de los Objetivos de Desarrollo del Milenio, pues, se encuentra un amplio abordaje en materia de desarrollo humano. La especificidad de aplicación de medidas para conservar la perspectiva regional y local, en cuanto a evitar el efecto de factores socioeconómicos y ambientales, esto se hace a través de la incidencia hacia la solidez y resiliencia de los sistemas de control de los determinantes sociales. No cabe duda de que el acceso a los servicios, entre ellos el de salud, tenga una importancia decisiva para el logro de cualquier adelanto sostenible en materia de potencialidad de desarrollo local, en especial en localidades cuya identidad cultural, incida sobre sus 
potencialidades de desarrollo económico local (Organización Panamericana de la Salud, 2017).

Como se observa, la necesidad de mejorar el efecto factorial de los determinantes sociales, entre ellos la salud, la cultura y la infografía permitirán que se logren cubrir las necesidades, y para ello, debe promoverse estrategias bajo el control de determinantes culturales, sociales, económicos, entre otros, estas consideraciones son un aspecto esencial del contexto Regional de las Américas, en la consecución de progresar y luchar frente indicadores de multiplicidad cultural, en esencia de la localidades de frontera.

Evidentemente, los desarrollos de las localidades, en especial de la región, se significan a razón de la competitividad económica, pues, se busca que en el futuro exista en todas las localidades del mundo transfronterizos un desarrollo para una vida plena que se manifieste en una horizontalidad hacia todos los seres humanos, en el entendido que los problemas globales son responsabilidad de todos los ciudadanos y como consecuencia o efecto la concienciación de lo más desarrollados.

Las economías mundiales tambalean cuando existen crisis globalizadas, por diferentes causas, y entienden de alguna manera, la importancia de apoyar a quienes realmente necesitan satisfacer sus necesidades, a pesar de poseer limitaciones de diferentes índoles. Cepal, ha argumentado en los diferentes informes las evidencias por las cuales, las estadísticas explanan el apoyo y aporte mundial de naciones desarrolladas, con cifras de miles de millones de dólares como asistencia oficial para el desarrollo, y se encuentran que las necesidades superan a los fondos entregados y se observa como el comercio internacional de 
bienes, ajusta las políticas proteccionistas de los países desarrollados (Donoso Molina \& García Vinueza, 2013).

En atención al impacto a una mayor sustentación local de las economías a países de pocos recursos, se generaliza el punto adicional, y no es menos que el haber un apoyo sobre las tecnologías del fututo para aumentar el desarrollo económico, pues, minimizarían las brechas digitales existentes en las poblaciones de economía mundial (Donoso Molina \& García Vinueza, 2013).

Además, se corregirían las faltas comunicacionales entre los pueblos más necesitados, ya es una cultura de identidad entre pueblos, y se sustentaría con mayor ahínco el desarrollo económico local, en especial en ciudades como las fronterizas en cuya esencia, la arquigrafia juega papel preponderante en las estrategias de competitividad.

Se observa en la actualidad que las instituciones financieras multilaterales se caracterizan por ser fuentes de financiamiento diverso en el desarrollo económico de las localidades, pero existe una nueva arquitectura en la esfera globalizada, y es la alianza para el desarrollo económico mundial, a través del financiamiento internacional, como principal fuente en proyectos y planes de desarrollo, y abarcaría un mayor crecimiento económico (Donoso Molina \& García Vinueza, 2013). Se infiere estas economías en desarrollo y emergentes dependerán cada vez menos de la asistencia económica internacional. 


\section{Conclusiones}

La identidad cultural de ciudades de fronteras, es una atribución representada por elementos del ser, cohesionados dentro de un grupo social en determinado territorio, en lo espacial y temporal. Recae sobre ellos, el sentimiento de pertenencia social, pero, con incidenciabilidad sobre las teorías antropológicas desde la perspectiva de la identidad cultural. La especificidad de las teorías está referida a las dinámicas internas de desarrollo de las sociedades e incidenciadas por la globalización respecto a valores con visión a excelencia operativa, liderazgo e intimidad del cliente. Entre otras teorías de la cultura se encuentran las materialistas como sistemas adaptativos entretejido del diseño de los componentes biológicos y culturales del humano, y las ideacionales como sistemas cognitivos culturas, estructurales y sistemas simbólicos.

Las ciudades y comunidades sostenibles son entes territoriales y cuando se hace referencia dentro del ámbito de la identidad cultural, encontramos que son parte altamente cosmopolitas sobre zonas de fronteras. La agenda 2030 persevera en el desarrollo sostenible y enfatiza que la economía mundial tiene que tener una dinámica inclusiva bajo la esfera de la heterogeneidad y el multiculturalismo, en especial en el contexto fronterizo, pues son pilares para fortalecer un resurgimiento económico poblacional local, con variabilidad, adaptabilidad, sostenibilidad y sustentabilidad.

La promoción de la identidad cultural, está vinculada con la arquigrafía para un potenciado económico en las ciudades de fronteras, es una 
herramienta para fortalecer la capacidad de competencia, que pudiese favorecer desde la proyección internacional. Ya estamos en conocimiento de que la globalización incide en la promoción de la identidad cultural en localidades fronterizas, por ello sobre las transformaciones sociales se concibe la estrategia competitiva como visión modal viable de ciudades de fronteras, la cual prevalece el enfoque de la productividad-país, con capacidades para un desarrollo económico existente de una localidad.

La estrategia competitiva de un territorio, es asumida como una ventaja sostenida de un sector derivado a la interacción que se generan por los determinantes de un sistema complejo de interacciones y evolución en el tiempo (Florez Ruiz, 2010). En contraste a este autor, allí encontramos los factores determinantes de la competitividad, configurados como sistema de relaciones, como se pudo expresar en el sistema completo del diamante de competitividad de Porter, la cual, se aprecian las múltiples relaciones entre los grupos de factores que lo integran según su teoría.

Una economía social eficaz y eficiente se genera cuando hay un impacto sobre el desarrollo territorial de ciudades de frontera, y ciertamente la estrategia competitiva se asienta en el uso de sus propios recursos endógenos que posea. Se puede inferir que los elementos actuantes del desarrollo local en el contexto de innovación y emprendimiento forman parte de las estructuras de las estrategias competitivas (Pérez González, 2010). En concordancia con esta autora, las estrategias radican en el propio carácter de las ciudades de frontera, a razón de sus realidades (territorial-necesidad-potencial 
endógena), el empleo y riqueza frente a recursos espaciales, las redes (agentes-territorios) deben priorizar los valores (sociales-principios básicos). Todo conjuga una visión modal viable de las ciudades de frontera.

El desarrollo económico local se formula como aquellas actuaciones entretejidas por agentes externos conllevados a cambios estructurales de un territorio (Barroso González \& Flores Ruiz, 2010). Infiriendo la postura de los autores, la finalidad que permite solventar la problemática, son el uso apropiado de recursos posibles desde los productivo, natural y humano.

El desarrollo económico local de ciudades de frontera, conlleva a sostener que la propia economía social es tratada singularmente (Pérez González, 2010). Conjugando el punto de vista del autor, los agentes dinamizadores intervinientes de un territorio son quienes a través de sus actuaciones impactan y multiplican dentro del ámbito espacial.

El desarrollo económico local y la economía social en el entorno espacial, ambas generan consecuencias que impactan de alguna manera sobre ciudades de fronteras, en ellas pesan la inserción laboral frente al tejido empresarial con actuación de flexibilidad frente a crisis temporales, por ejemplo (Caso COVID-19). Allí, se destaca la revalorización de activos locales para una calidad de vida (Caso ciudades fronterizas nortesantandereana y tachirense), la cohesión y transformaciones sociales, y finalmente los valores medioambientales y culturales en el ámbito desarrollo territorial.

La competitividad y estimulación de empresas y mercados incidenciadas en el desarrollo económico local de las ciudades de 
fronteras, hace que el proceso embargue la caracterización del tejido empresarial. La sostenibilidad en materia de identidad cultural como base estratégica competitiva se enfoca con visión a economías desarrolladas en localidades con entornos departamentales entre países vecinos. Están regiones manejan el empleo y generan aceleradas economías.

El desarrollo como objetivo del milenio, se justifica por que conlleva a una mejora de la vida de todas las personas en las localidades del mundo, fronterizas o no fronterizas, y solo se logrará a través de las economías saneadas y la satisfacción de las necesidades de los ciudadanos con una previa asistencia de los países desarrollados, que apoyan a los de menos recursos; pero, entendiendo que el trabajo debe ser cooperativo y conjunto, bajo la interacción multidisciplinaria en el ámbito global, regional, nacional y local, dando prioridades a intereses comunes entre organizaciones y financiadores (Arévalo Ramos, 2016), (Villasmil Jiménez \& Romero Prada, 2011).

Por último, se hace referencia a las situaciones fácticas acaecidas en el mundo por el caso incidencial del COVID-19, cuya incidencia trae cifras alarmantes producto de la pandemia ejemplarizado en (Dinero.com, 2020). Un desempleo en naciones como EE. UU, que alcanzó los 38 millones, afectando la economía, con pérdidas semanales sistemáticas. Colombia ha sido afectada por el duro panorama económico con un escenario en el mercado laboral de por lo menos 6 millones de desempleo e inactividad, representan el $12,6 \%$. Se infiere, que el impacto por desocupación es un problema 
estructural en productividad, y desarrollo económico de localidades como las fronterizas.

\section{Referencias}

Agenda para el Desarrollo Sostenible. (30 de enero de 2020). Objetivo 11. Ciudades y comunidades sostenibles. Obtenido de Objetivos del desarrollo sostenible de la Agenda 2030.: https://www.jointsdgfund.org

Arévalo Ramos, C. (2016). Objetivos de Desarrollo del Milenio. Guatemala: Universidad Rafael Landivar.

Aste, E. (s/f). Reingeniería enfocada al cliente. Obtenido de Revistas. blse.aste. Año 2. Nro.4: http://200.16.86.50/digital/33/revistas/blse/aste1-1.pdf

Ballesteros, C., Gracia, G., Ocaña, A., \& Jácome, C. (julio-diciembre de 2018). Análisis de la promoción cultural como herramienta para fortalecer la identidad afro esmeraldeña1. (C. U. Lasallista, Ed.) Revista Lasallista de Investigación, 15(2), 367-377. Obtenido de https://www.redalyc.org/jatsRepo/695/69559233029/69559233029.pdf

Bárcena, A. (13 de diciembre de 2019). CEPAL: América Latina cerrará en 2020 su peor período económico en 70 años. Obtenido de Negocios y Economía: https://www.voanoticias.com/economia-finanzas/cepal-america-latina-cerrara-su-peorperiodo-economico-en-2020

Barroso González, M., \& Flores Ruiz, D. (2010). Teoría y estrategias de desarrollo local. Sevilla.España: Universidad Internacional De Andalucía.

Del consultores, desarrollo económico, planificación estrategica y emprendimiento. ( $\mathrm{s} / \mathrm{f}$ ). Estrategias de desarrollo económico. Obtenido de ¿Qué es Desarrollo Económico Local?: http://www.delconsultors.com/es/que-es-del/

Donoso Molina, M., \& García Vinueza, E. (2013). Instituto Superior de Postgrado en Ciencias Internacionales. "Dr. Antonio Parra Velasco". M. Instituto Superior de Postgrado en Ciencias Internacionales. "Dr. Antonio Parra VeTesis presentada como requisito para obtener el Grado de Magíster en Negociación y Comercio Internacional. Guayaquil. Ecuador: Instituto Superior de Postgrado en Ciencias Internacionales. "Dr. Antonio Parra Velasco".

DW.Español. (21 de mayo de 2020). El Mundo. Actualidad Política. Obtenido de Coronavirus, minuto a minuto: pandemia avanza en América Latina con sombrío pronóstico: https://www.dw.com/es/coronavirus-minuto-a-minuto-pandemia-avanza-enam\%C3\%A9rica-latina-con-sombr\%C3\%ADo-pron\%C3\%B3stico/a-53527369

Enguix, B. (2010). Cultura, culturas, antropología. Obtenido de Universidat Oberta de Catalunya: http://creativecommons.org/licenses/by-nc-nd/3.0/es/legalcode.es.

Flores $\mathrm{H}$, I. (2005). Identidad cultural y el sentimiento de pertenencia a un espacio social. Obtenido de Una discusión teórica: https://cdigital.uv.mx/bitstream/handle/123456789/345/2005136P41.pdf?sequence= $1 \&$ is

Flores Hernández, E. (10 de diciembre de 2013). Territorios y Estrategia Competitiva. 
Programa ConectaDEL a través de Fundación Demuca en Centroamérica., 1-17. Obtenido de Universidad Centroamericana "José Simeón. ConectaDEL. Programa para la Formación en Desarrollo Económico Local con Inclusión Social en América Latina y El Caribe.

Florez Ruiz, D. (2010). Competitividad territorial y sistemas productivos locales. capítulo V. En M. y. Barrozo Gonzalez, Teoria y estrategia de Desarrollo Local (págs. 139-169). Sevilla. España: Universidad de Huelva.

García Nicolás, C. (30 de noviembre de 2015). Competitividad y territorios de frontera: retos ante el plan. Obtenido de https://www.researchgate.net/publication/285131825

Observatorio de Desarrollo Local. (20 de enero de 2020). V foro mundial de desarrollo económico local 2020. Obtenido de ConectaDel: http://www.conectadel.org/v-foromundial-de-desarrollo-economico-local-2020/

OMS. (27 de febrero de 2020). Prepare su lugar de trabajo para la COVID-19. Obtenido de https://www.paho.org/es/documentos/prepare-su-lugar-trabajo-para-covid-19

ONU. (2019). Situación perspectivas de la económia mundial. Obtenido de https://www.un.org/development/desa/dpad/wpcontent/uploads/sites/45/publication/WESP2020_Summary_S.pdf.

Organización de las Naciones Unidas para la Alimentación y Agricultura. (2020). Objetivos de Desarrollo Sostenible. Recuperado el 04 de junio de 2020, de Objetivo de Desarrollo del Milenio 8: Fomentar una asociación mundial para el desarrollo: http://www.fao.org/sustainable-development-goals/mdg/goal-8/es/

Organización Mundial de la Salud. (2020). Objetivos de Desarrollo del Milenio. Recuperado el 30 de mayo de 2020, de https://www.who.int/topics/millennium_development_goals/about/es/

Organización Panamericana de la Salud. (2017). Informe final sobre los Objetivos de Desarrollo del Milenio relacionados con la salud en la Región de las Américas. Recuperado el 30 de mayo de 2020, de https://iris.paho.org/bitstream/handle/10665.2/34114/9789275118782_spa.pdf?sequ ence $=5$ \&isAllowed $=y$

Pérez González, M. (2010). Desarrollo Local y Economía Social. Capítulo IX. En M. Barroso González, \& D. Florez Ruíz, Teoría y Estrategias Desarrollo Local (págs. 271-308). Sevilla. España: Universidad de Cádiz.

Publinews. (29 de 05 de 2020). Coronavirus avanza en América Latina con sombrío impacto para sus economías. Obtenido de Noticias. Coronavirus avanza en América Latina : https://www.publinews.gt/gt/noticias/2020/05/22/coronavirus-avanza-en-americalatina.html

Rhi-Sausi, J., \& Conato, D. (2009). Cooperación transfronteriza e integración en américa latina:la experiencia del proyecto fronteras abiertas. España: Proyecto IILA-CesPI. Obtenido de Proyecto Fronteras Abiertas.

Rodríguez, I. (octubre de 2000). Heterogeneidad y multiculturalismo.¿discusión cultural o discusión legal? Revista Iberoamericana. , LXVI(193), 851-861.

Sociedad Geográfica de Colombia. (2006). Horizonte futuro de las Fronteras: Globalización . Obtenido 
https://sogeocol.edu.co/Ova/fronteras_colombia/documentos/fronteras_pasadoprese ntefuturo.pdf

TINA Viena, Departamento Municipal 18. (2016). Desarrollo y planificación de la ciudad (2da. Edición ed.). (A. d. Viena, Ed.) Viena: Administración de la Ciudad de Viena.

Vergara Estévez, J., \& Vergara D., J. (2002). Cuatro tesis sobre la identidad cultural latinoamericana una reflexión sociológica. (U. A. Tarapacá, Ed.) Revista de Ciencias Sociales (Cl)(12), 77-92.

Villasmil Jiménez, L. C., \& Romero Prada, J. R. (enero-junio de 2011). Los objetivos de desarrollo del milenio de las naciones unidas: ¿en dónde estamos y para dónde vamos? Lasallista de Investigación, 8(1), 126-135. 

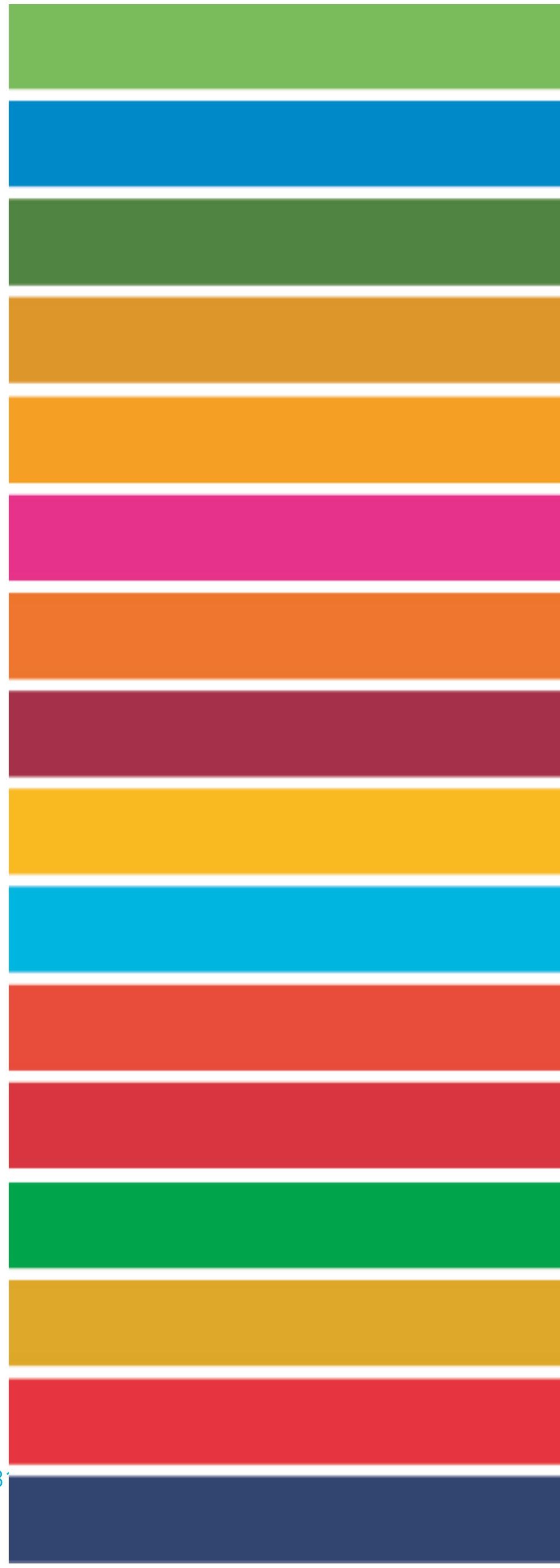